\title{
Assessing Underwater Visibility Conditions in a Large River
}

\author{
Alexander Anatol ERMILOV ${ }^{凶}$, Flóra POMÁZI, and Sándor BARANYA \\ Department of Hydraulic and Water Resources Engineering, \\ Budapest University of Technology and Economics, \\ Budapest, Hungary \\ ermilov.alexander@emk.bme.hu
}

\begin{abstract}
In this study, the comparison of measured suspended sediment concentrations, calculated light transmissivity and underwater images was done in order to assess the visibility conditions in a large river and to investigate the opportunities of a possible forecasting methodology that could support diving operations and underwater image processing methods. Further discussion will be made based on the first set of results. The measurements were carried out in the Hungarian section of river Danube.
\end{abstract}

Keywords: suspended sediment, river, transmissivity, underwater camera.

\section{INTRODUCTION}

Underwater diving operations in rivers are always hazardous due to the limited visibility conditions and strong currents. Hence, pre-monitoring of the diving sites in terms of physical conditions is essential. Acoustic Doppler Current Profiler (ADCP) measurements not only give information about the flow velocities, but the suspended sediment concentration (SSC) can also be assessed (Pomázi and Baranya 2020), playing an important role in visibility. Moreover, SSC values can provide the so-called optical transmissivity parameter (i.e. the proportion of visible light transmitted through unit distance of the turbid medium) (McCarthy et al. 1974). This could give information for the divers about visibility distance. In this study, we used ADCP measurements to estimate SSC and optical transmissivity in a case study in the Hungarian Danube.

Nowadays, computer vision and image-processing methods are rapidly improving and spreading into more and more field of engineering, for instance, hydraulic engineering. In our earlier study of river Danube (Ermilov et al. 2020), underwater cameras were deployed on the 
field to take images of the riverbed and bedload to quantify grainsize distributions and bedload transport rate. These images were used here to validate the calculated optical transmissivities.

\section{METHODOLOGY}

In a recent study (Pomázi and Baranya 2020), we tested a $1200 \mathrm{kHz}$ ADCP to derive SSC distributions from acoustic backscatter. Time-averaged echo intensity (EI) profile (Fig. 1a) could be determined from fixed-boat ADCP measurement. We performed the calibration by the socalled sonar equation (Gartner 2004), establishing a relationship $\left(R^{2}=0.60\right)$ between the relative backscatter and the measured SSC. An example for the calibrated SSC profile is shown on Fig. 1b. Additionally, we analysed the above-mentioned physical samples with the LISSTPortable|XR laser diffraction device applying the Mie-theory for quartz particles. Besides SSC, laser diffraction provides particle size distribution of the suspended sediment in the 0.34$500 \mu \mathrm{m}$ size range, as well as the optical transmission (\%) of the samples. Based on the relationship between the SSC and the optical transmission data, we estimated the vertical variation of transmissivity due to suspended sediment particles (Fig. 1c).

When measuring the sediment conditions along the water columns with ADCP and physical samplings, a camera was also lowered from the measurement vessel. The footages here were used to check the colour range of the infiltrating natural light, depending on the water depth and SSC. The changes in the colour are clearly visible in Fig. 1d. We found, that the boundaries are related to the inflexions of the optical transmission profile. Besides, estimation of visibility distances could also be done to validate the calculated optical transmissivity.
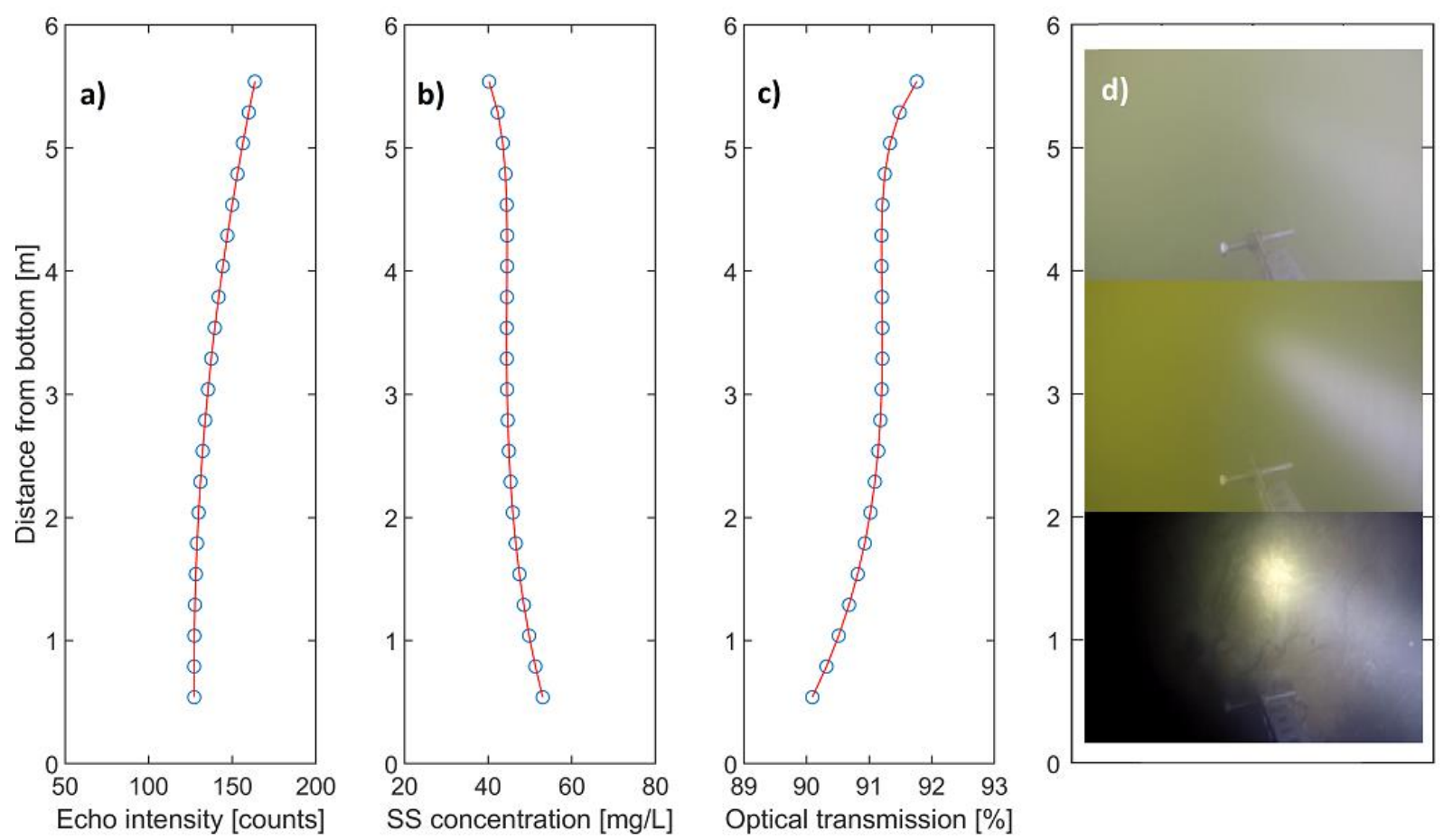

Fig. 1: a) Echo intensity profile, b) calibrated SSC profile, c) optical transmission along the vertical, d) example of images from one vertical, taken during the lowering of the camera (top: close to the water surface, bottom: riverbed). 


\section{References}

Ermilov, A.A., S. Baranya, and G.T. Török (2020), Image-based bed material mapping of a large river, Water 12, 3, 916; DOI: 10.3390/w12030916.

Gartner, J.W. (2004), Estimating suspended solids concentrations from backscatter intensity measured by acoustic Doppler current profiler in San Francisco Bay, California, Mar. Geol. 211, 3-4, 169-187, DOI: 10.1016/j.margeo.2004.07.001.

McCarthy, J.C., T.E. Pyle, and G.M. Griffin (1974), Light transmissivity, suspended sediments and the legal definition of turbidity, Estuarine Coast. Mar. Sci. 2, 3, 291-299, DOI: 10.1016/03023524(74)90019-X.

Pomázi, F.; and S. Baranya (2020), Comparative assessment of fluvial suspended sediment concentration analysis methods, Water 12, 3, 873, DOI: 10.3390/w12030873.

Received 22 March 2021

Accepted 12 April 2021 